\title{
NOTAS TEXTUALES Y PROSOPOGRÁFICAS SOBRE PLIN., NAT. XXIX 7-8
}

\author{
Marc MaYer i Olivé \\ Universidad de Barcelona \\ mayerolive@yahoo.es
}

\section{TEXTUAL AND PROSOPOGRAPHIC NOTES ON PLIN., NAT. XXIX 7-8}

El pasaje de Plinio el Viejo (Nat. XXIX 7-8) permite a través de una nueva fijación del texto comprobar noticias sobre el médico del emperador Claudio Stertinius Xenophon hasta ahora sólo supuestas.

Palabras clave: Crítica textual; Literatura latina; Historia de Roma
Thanks to a new reading of the text, the passage from Pliny the Elder (Nat. XXIX 7-8) confirms some data about the physician of the emperor Claudius Stertinius Xenophon which so far has been only object of conjectures.

Keywords: Textual criticism; Latin literature; Roman history.

Un pasaje de Plinio sobre la importancia y los salarios de los médicos en Roma plantea cuestiones que van mucho más allá del valor de simple anécdota, teñida incluso de crítica social, que se le ha querido dar al pasaje ${ }^{1}$ :

Multos praetereo medicos celeberrimosque ex iis Cassios, Carpetanos, Arruntios, Rubrios. $\bar{C} \bar{C} \bar{L}$ HS annuae iis mercedes fuisse apud principes. Q. Stertinius inputauit principibus, quod sestertiis quingenis annuis contentus esset, sescena sibi quaestu urbis fuisse enumeratis domibus ostendebat. Par et fratris eius merces a Claudio Caesare infusa est, censusque, quamquam exhausti operibus Napoli exornata, heredi HS $\bar{C} \bar{C} \bar{C}$ reliquere, quantum aetate eadem Arruntius solus.

Los problemas del texto son múltiples y casi todos ellos tienen una connotación prosopográfica importante. El primero de los problemas va ligado

${ }^{1}$ Nos servimos de la edición de L. Ian y C. Mayhoff, C. Plinii Secundi naturalis historiae libri XXXVII, vol. IV: Libri XXIII-XXX, Leipzig, 1897, p. 370. 
a la identidad del $Q$. Stertinius presente en el pasaje pliniano, y el segundo a la cuantía del legado.

El personaje citado ha sido objeto de sucesivas aproximaciones y por nuestra parte adelantamos ya que creemos que debe tratarse del médico de Claudio, G. Stertinius Xenophon, aunque esta identificación sea negada por algunos repertorios en los últimos tiempos.

De ser cierta esta identificación, el hermano mencionado podría no ser otro que el tribuno militar de la legión XXII Primigenia, Tiberius Claudius Cleonimus, que tenemos documentado como veremos más adelante. Éste, de acuerdo con las tradiciones familiares, sería también médico. Por otra parte, es conocida la protección ejercida por Gayo Estertinio Jenofonte sobre su familia y el hecho de que los Asklepiadai de la isla de Cos, a cuyo núcleo pertenecen los dos personajes, tuvieron una larga y conocida trayectoria como médicos ${ }^{2}$.

Los problemas, en lo que concierne a la hipótesis de identificación del personaje citado por Plinio el Viejo, se basan en la evidente discordancia que presenta el praenomen transmitido en el texto comúnmente aceptado, Quinto, con el de Gayo Estertinio Jenofonte, cuestión para la cual resulta indispensable recurrir al aparato crítico del texto con el fin de verificar la solidez y fiabilidad de la lectura.

El praenomen abreviado $Q$. está presente solamente en $\mathrm{R}$ (el codex Florentinus Riccardianus, de indudable autoridad) y en $S$ (la edición de Silligius [Hamburgo, 1855]), mientras que la lectura de $B$ (Hermolao Bárbaro en sus Castigationes Plinianae [Roma, 1492, 1493]) establece la lección Q. uero; por su parte, $r, v$, es decir, la lectio vulgata, y todos los demás códices, entre los cuales se cuentan V (codex Leidensis Vossianus fol. $n$. LXI, de indudable importancia), d (codex Parisinus latinus 6797) y E (codex Parisinus latinus 6795) dan la lección quae. La cuestión, por consiguiente, no es fácil, y aunque aparentemente la $q$ inicial parezca segura en todas las versiones del texto, no resulta tan claro que sea la abreviación de un praenomen. La lectura únicamente como $Q$. parece responder a una simplificación de un texto que podía resultar difícilmente comprensible. En nuestra opinión, no obstante, la lectura quae quizá podría resolver el problema, ya que recogería, con un

2 S. M. Sherwin-Withe, Ancient Cos. An historical study from the Dorian settlement to the Imperial period, Gotinga, 1978, pp. 149-152, hace un completo resumen sobre este miembro de la familia de los Asklepiadai de Cos y su implicación en su isla de origen. 
relativo ilativo, lo dicho anteriormente respecto a los otros médicos, y, por consiguiente, la mención de Stertinius podría no especificar como en los demás casos el praenomen. La forma propuesta por Hermolao Bárbaro Q. uero procedería, en consecuencia, de un intento de intelección de quae como una $q$, posiblemente seguida de punto, y de una abreviación para uero, intentando resolver la dificultad mediante el mantenimiento, en lo posible, del número de letras. En este mismo sentido se pronunció en su momento R. Herzog, que supuso la lectura quae Stertinius, propuesta aceptada recientemente por V. Nutton $^{3}$. No obstante, lo que resulta importante constatar es que el texto tiene problemas suficientes como para poder pensar en una corrupción, y que, si se tiene en cuenta lo poco frecuente del nomen Stertinius y el contenido del texto, deberemos convenir que, como mínimo, existe una alta probabilidad de que el personaje pliniano y el médico de Claudio, documentado en los textos de Tácito y también abundantemente por la epigrafía, sean la misma persona. Hemos de sumar a ello, además, que en el caso de Estertinio Jenofonte tenemos bien probada la existencia de un hermano.

Una segunda cuestión pende sobre este pasaje: el legado de ambos médicos y su montante. La suma del legado hereditario resulta de explicación más simple si atendemos a las variantes textuales. El texto admitido generalmente es $H S \bar{C} \bar{C} \bar{C}$, es decir, trescientos mil sestercios, aunque muy probablemente el montante fuera, como se acepta generalmente, por el propio énfasis de la explicación de Plinio, treinta millones ${ }^{4}$.

El aparato crítico debe venir de nuevo en nuestra ayuda. La forma $H S$ $C C C$ es la propuesta por $H$, es decir, Harduinus en su edición de París de 1685. Los manuscritos $\mathrm{E}$ y $\mathrm{r}$, que representa las correcciones a $\mathrm{R}$ según un manuscrito que nos es desconocido, así como la lectio vulgata y la edición de Silligius, que la sigue, indican $H S C C C$. El manuscrito d contiene la lección hs. $u$. CCCI, y el códice V, sti. ceci, ambas lecturas con una evidente falta de comprensión del significado. Evidentemente se trata de treinta millones

${ }^{3}$ R. Herzog, «Nikias und Xenophon von Kos. Zwei Charakterköpfen aus der griechischrömischen Geschichte», $H Z$ 125, 1922, pp. 189-247, esp. p. 229, n. 1; V. Nutton, «Roman Medicine: Tradition, Confrontation, Assimilation», en $A N R W$ II 37.1, Berlín-Nueva York, 1993, pp. 49-78, esp. p. 63, n. 63.

${ }^{4}$ R. Duncan-Jones, The Economy of the Roman Empire. Quantitative Studies, Cambridge, 1974, pp. 343-344, para una lista inicial de fortunas privadas; esp. núm. 19, p. 344, para la que nos ocupa. No menciona este autor, en cambio, la fortuna equivalente del médico Arruntius, citado como comparación en el mismo texto. 
y, por consiguiente, de $H S I \bar{C} \bar{C} \bar{C} I$. La clave nos viene dada precisamente por las lecturas de $\mathrm{d}$ y de $\mathrm{V}$ : resulta claro que en ambos casos la I final es la barra multiplicadora del numeral. En el caso de d la forma $h s$. $u$. podría ocultar en $u$ la primera barra multiplicadora o bien incluso una posible $N$ de $n$ (uтmum), como se indican a menudo las cantidades monetarias sobre todo en las inscripciones. Más evidente todavía puede resultar V, donde sti. puede contener la indicación de $H S$ y la primera barra multiplicadora $I$; además cabe pensar incluso que la $t$ pudiera ser un resultado de la confusión de lectura de la supralineación del numeral. La última barra es manifiesta en la forma ceci por CCCI. Parece claro que la explicación textual asegura una lectura $I \bar{C} \bar{C} \bar{C} I$, frente al texto generalmente aceptado, y que la cuantía del legado es de treinta millones de sestercios como, por otra parte, siempre han supuesto los estudiosos conocedores de la economía romana, corrigiendo tan sólo el contenido del texto, sin entrar en él en razón de los montantes conocidos para este tipo de herencias. Parece claro, en consecuencia, que se trata de una corrupción en la tradición textual que, no obstante, deja entrever, si se presta la debida atención y teniendo en cuenta los precedentes, la lectura original. Errores de copia o de transmisión de este tipo son además muy frecuentes en las inscripciones perdidas de tradición literaria, lo cual refuerza una interpretación como la que proponemos sobre la base de la crítica del texto.

Adquirido ya un resultado, nos parece que con visos importantes de fiabilidad, para este segundo problema, podemos volver al primero, para intentar aducir un razonamiento histórico a la propuesta de identificación del personaje que ya hemos adelantado.

En primer lugar, creemos que deben descartarse las soluciones prosopográficas mixtas propuestas hasta ahora, como la de R. Duncan-Jones, que, tomando como base el texto de Plinio, admite la existencia de $Q$. Stertinius y supone que el otro hermano no explícitamente mencionado sería el famoso C. Stertinius Xenophon, médico de los emperadores 5 .

Aunque es generalmente aceptada por los historiadores la identificación entre el personaje mencionado explícitamente por Plinio y el famoso médico de Claudio C. Stertinius Xenophon ${ }^{6}$, existen sin embargo discrepancias,

${ }^{5}$ Duncan-Jones, The Economy of the Roman Empire..., p. 344.

${ }^{6}$ Fue médico de Tiberio, de Calígula, de Claudio y de Nerón. Cf. F. Kudlien, Die Stellung des Arztes in der römischen Gesellschaft. Freigeborene Römer, Eingebürgerte, Peregrine, Sklaven, Freigelassene als Ärtze, Stuttgart, 1986, pp. 57-60. Cf. también el apéndice «C. 
especialmente en algunos de los estudios prosopográficos recientes que han tendido a confiar en el texto recibido de Plinio y a separar ambos personajes sin tener en cuenta las dificultades de tradición textual del pasaje sobre el que se basaban sus hipótesis?

Por nuestra parte, como ya hemos adelantado, estamos convencidos de que se trata del mismo personaje que fue médico de Claudio, y no de un

Stertinius Xenophon und sein Haus», en R. Herzog, Koische Forschungen und Funde, Leipzig, 1899, pp. 189-199; V. Nutton, «Archiatri and the medical profession in antiquity», PBSR 45, 1977, pp. 191-226, esp. pp. 195-196; J. Korpela, Das Medizinalpersonal im antiken Rom. Eine sozialgeschichtliche Untersuchung, Helsinki, 1987, núm. 115, p. 175; G. Marasco, «I medici di corte nella società imperiale», Chiron 28, 1998, pp. 267-285, esp. pp. 268-270 y 275-277; íd., «I medici di corte nell'impero romano: prosopografia e ruolo culturale», Prometheus 24, 1998, pp. 243-263, esp. pp. 257-258, núm. 41; íd., «Medici alla corte dei Cesari: Funzioni e metodi terapeutici», Medizinhistorisches Journal 32, 1997, pp. 279-297, esp. pp. 290-291; íd., «Les salaires des medicins en Grèce et à Rome», en Le normal et le pathologique dans la Collection hippocratique. Actes du IX'me colloque international hippocratique (Nice: 6-9, X, 1999), Niza, 2002, pp. 769-786; K. Buraselis, Kos Between Hellenism and Rome. Studies on the Political, Institutional and Social History of Kos from ca. the Middle Second Century B.C. until Late Antiquity, Filadelfia, 2000, pp. 66-110. En general para el significado de este cursus véase G. Alföldy, «Die Stellung der Ritter in der Führungsschicht des Imperium Romanum», Chiron 11, 1981, pp. 169-215, con un excelente y claramente formulado estado de la cuestión, y especialmente en p. 190, n. 104, donde se inclina por considerar el mismo personaje al citado por Plinio y al de las menciones epigráficas; así lo hace también F. Millar, The Emperor in the Roman World (31 BC-AD 337), Ithaca, 1977, pp. 85-86, que cree que Claudio contrató a C. Estertinio y a su hermano por la suma de quinientos mil sestercios, y parece suponer que Gayo Estertinio sería el citado por Plinio, Nat. XXIX 7-8.

7 PIR 2 S 913, pp. 337-340, con árbol genealógico en p. 340, donde no es considerado la misma persona que el médico citado por Plinio, que embelleció Nápoles y dejó junto con su hermano una herencia de treinta millones de sestercios; cf. PIR ${ }^{2} \mathrm{~S} 905$. Q.(?) Stertinius y anteriormente PIR III, S 658, p. 272, para este posible Q. Stertinius. Vease, además, S. Demougin, Prosopographie des chevaliers romain julio-claudiens (43 av. J.-C. - 70 ap. J.-C.), Roma, 1992, núm. 487, pp. 396-397, y esp. p. 397, donde hace una diferenciación entre los dos Estertinios y precisa también que Philinus era su tío materno y no paterno, como admite también $P I R^{2}$. Acepta la existencia de un médico diverso con este nombre Marasco, «I medici di corte nell'impero romano», p. 258, núm. 42, donde recoge sin embargo las opiniones discrepantes, en especial la de D. Gourevitch, Le triangle hippocratique dans le monde grécoromain. Le malade, la maladie et son médicin, Roma, 1984, p. 351, n. 13, que señala las dudas sobre la existencia de Q. Stertinius, y de Nutton, «Roman Medicine...», p. 63, n. 63, que se pronuncia por un error en el texto de Plinio; sin embargo Korpela, Das Medizinalpersonal..., núm. 114, p. 175, cree también en la existencia de dos personajes distintos siguiendo el texto comúnmente aceptado de Plinio. 
pariente, ni siquiera de un hermano, como pretenden algunas propuestas de identificación.

Un breve excurso sobre su familia, biografía y carrera puede resultar útil para encuadrar el problema. El personaje, Gaius Stertinius Xenophon hijo de Heráclito, de la tribu Cornelia, es definido por los epígrafes que lo honran en

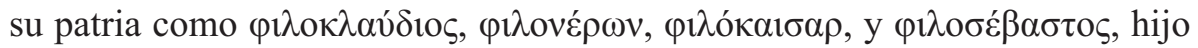

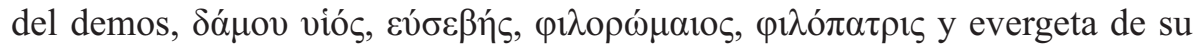

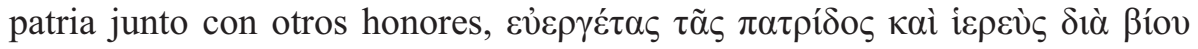

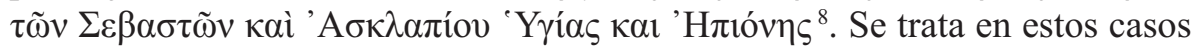
evidentemente del famoso médico del emperador Claudio, y anteriormente de Tiberio. Estertinio Jenofonte siguió paralelamente a su función como médico, y como consecuencia de ésta, un cursus ecuestre ${ }^{9}$. Conocemos bien sus lazos familiares y también la protección que parece haber ejercido sobre sus pa-

${ }^{8}$ M. Segre, Iscrizioni di Cos, Roma, 1993, núms. EV 22, 25, 43, 46, 68, 70, 83, 85, 95, $97,112,117,119,124,126$ ? $, 128,143,205,219$, pedestal de estatua situado junto al famoso plátano de Cos, 221, 233, 237?, 241, 245, 286, 288, 289, 290?, 294?, 295?, 296, 297, 298, 299, 300?, 301, 302, 311, 314, 315, 316, 317, 318, 319?, 320?, 321, 322?, 324?, 325, 327, 337, 341, 344?, 347, 365, 366; véase ahora el índice onomástico en M. Segre, Iscrizioni di Cos, vol. 2, Roma, 2007, p. 221; A. Maiuri, Nuova Silloge epigrafica di Rodi e Cos, Florencia, 1925, pp. 173-177, esp. núms. 475- 478, pp. 174-175, y núm. 485, quizá de nuevo Estertinio, p. 178, n. ${ }^{\circ}$ 585; W. R. Paton y E. L. Hicks, The Inscriptions of Cos, Oxford, 1891, núms. 84-94, pp. 127-132; Herzog, Koische Forschungen und Funde, núms 21-25, pp. 64-66. Un resumen en $P I R^{2}$ S. 913, pp. 337-338, con las numerosas novedades pasadas a la redacción, en este caso a cargo de K. Wachtel, por K. Hallof. Véase además J. Benedum, «Inschriften aus Kos», ZPE 27, 1977, pp. 229-240, esp. pp. 239-240, con tres ejemplares con el mismo contenido (dos de ellos dados como inéditos en la edición de Segre: n. $^{\circ} 6=$ EV 302, n. ${ }^{\circ} 7=$ EV 83). Véase, finalmente, É. Samama, Les médecins dans le monde grec. Sources épigraphiques sur la naissance d'un corps médical, Ginebra, 2003, pp. 257-263 (n. $\left.{ }^{\text {os }} 142-147\right), 269$ (n. $\left.{ }^{\circ} 154\right)$ y $558-561$.

${ }^{9}$ Para su cursus cf. H.-G. Pflaum, Les carrières procuratoriennes équestres sous le Haut-Empire romain, vol. 1, París, 1960, núm. 16, pp. 41-44. Syll $804=S y l l^{2}$ 369s recogen su cursus, y en especial su cargo ab epistulis Graecis o encargado de la sección ad responsa Graeca, en su formulación griega, que parece haber culminado su carrera ecuestre. Sobre el personaje y su cursus véase también PIR III S 666, pp. 273-274; H. Devijver, Prosopographia militiarum equestrium quae fuerunt ab Augusto ad Gallienum, Pars secunda. Litterae L - V, Ignoti - Incerti, Lovaina, 1977, núm. S 79, pp. 758-759; A. Stein, Der römische Ritterstand. Ein Beitrag zur Sozial- und Personengeschichte des römischen Reiches, Múnich, 1927, pp. 186-187 y 400; S. Mratschek-Halfmann, Divites et praepotentes. Reichtum und soziale Stellung in der Literatur der Prinzipatzeit, Stuttgart, 1993, núm. 101, pp. 297-298, que ve las dificultades textuales de la identificación. 


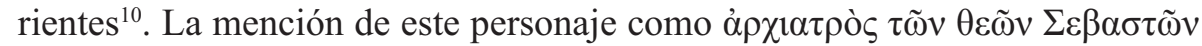
de la inscripción de Calymnos es importante para nuestro objetivo, que es fijar su significación ${ }^{11}$.

El nombre del médico presenta una evidente homonimia con el cónsul sufecto del año 23 d. C., C. Stertinius Maximus, precisamente el año en el que una embajada de Cos comparecía ante el senado romano para defender el ius asyli del santuario de Asclepio. El nombre Estertinio ha hecho suponer que Jenofonte no sólo obtuvo el privilegio pedido para el santuario, sino que también logró, con la protección del cónsul, la ciudadanía romana probablemente en aquel mismo momento, ya que seguramente habría formado parte de la delegación que la isla de Cos mandó a Roma en su condición previa de peregrinus $^{12}$. De aquí, además, la diferencia onomástica con su hermano, Tiberius Claudius Cleonimus, hijo de Heráclito, de la tribu Quirina, que debió de recibir más tarde su privilegio directamente del emperador ${ }^{13}$.

Las al menos casi sesenta inscripciones que le son dedicadas parecen datar todas del siglo I d. C. Fue tribuno militar a los cincuenta años, y fue condecorado bajo Claudio en la expedición británica ${ }^{14}$. Parece, no obstante, que

${ }^{10}$ Cf. Syll ${ }^{3} 805$, pp. $498-499$ (= Syll 369 ), para su hermano que se llama Tiberio Claudio Cleónimo, hijo de Heráclito de la tribu Quirina, tribuno de la legión XXII Primigenia en Germania, esposo de una Claudia Phoibe; Syll 806, p. 499 (= Syll 370 ), para Tiberio Claudio Filino hijo de Jenofonte, tío materno de Gayo Estertinio Jenofonte; Segre, Iscrizioni di

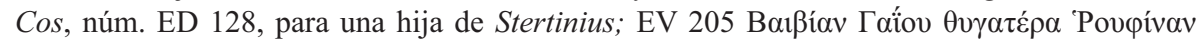
esposa de Stertinius; núm. 233 corresponde al hermano militar de Xenophon; núm. 237 es seguramente un pariente. H. Devijver, Prosopographia militiarum equestrium...; núm. C 134 para Ti. Claudius Cleonymus y C 166 para Ti. Claudius Philinus.

${ }^{11} S_{y} l l^{3}$ 804, pp. 496-498 (= Syll 368, Paton y Hicks, The Inscriptions of Cos, núm. 345), con mención de sus condecoraciones, corona aurea y hasta pura, en la campaña británica.

${ }^{12}$ Cf. para C. Stertinius Maximus PIR² S 908, p. 336. R. Syme, «Problems about Proconsuls of Asia», Roman Papers, IV, Oxford, 1988, pp. 347-365, (anteriormente ZPE 53, 1983, pp. 191-208), esp. pp. 357-358, piensa que Estertinio Jenofonte obtuvo su ciudadanía en el decurso de alguna función de Gayo Estertinio Máximo en Asia. Cf., además, E. Birley, «Septimius Severus and the Roman Army», Epigraphische Studien 8, 1969, pp. 63-82, esp. p. 80. Devijver, Prosopographia militiarum equestrium..., p. 759 , se inclina también por una concesión de la ciudadanía por parte del magistrado en Cos.

${ }^{13}$ Cf. nota 8. Quizá Tiberio Claudio, de la tribu Quirina, Menécrates, que fue médico de Nerón, pueda pertenecer a la misma familia, cf. Syll 367 . Sobre este personaje cf. Korpela, Das Medizinalpersonal..., núm. 70, pp. 167-168, para el que propone también concomitancias con el Trimalción de Petronio.

${ }^{14}$ Cf. nota 7. 
sobre él pesa la sospecha de haber sido quien, por instigación de Agripina, envenenó al propio emperador que le había colmado de honores ${ }^{15}$, aunque en esta cuestión no hay acuerdo ${ }^{16}$. El personaje ha sido comparado, además, con la figura literaria de Trimalción — sobre todo si se le puede identificar, como proponemos, con el personaje citado por Plinio el Viejo-, tanto por sus bienes ${ }^{17}$, por su sueldo extraordinario de quinientos mil sestercios, como por la herencia legada junto con su hermano de treinta millones de sestercios ${ }^{18}$.

Resulta claro que en una obra erudita que ve la luz en el período Flavio como la Historia natural de Plinio el Viejo, Estertinio Jenofonte no necesita presentación: se trata de un personaje tópico, característico de una cierta profesión y con una intervención nada anecdótica en el reinado de Claudio y que, como la gran mayoría del entorno de este emperador, acumuló una ingente fortuna.

Estas razones nos parecen suficientes para que, a la vista del estado del texto de Plinio, pueda abrirse una duda razonable sobre la identidad real del Q. Stertinius recogido en el pasaje que estudiamos y sobre su existencia, considerada por algunos estudiosos como indiscutible a partir de su mención en él. Más allá de suponer un error de Plinio respecto al praenomen, si queremos admitir la identificación con C. Stertinius Xenophon, como no ha dejado de suponerse en la tradición historiográfica que admite este hecho, podemos pensar en una corrupción del texto que ha conducido a una lectura que ha creado un pretendido $Q$. Stertinius.

15 Tac., Ann. XII 61, para la importancia de la familia de Estertinio Jenofonte, y II 67 para su participación en el asesinato de Claudio, cf. Herzog, «Nikias und Xenophon von Kos...», pp. 189-247, esp. pp. 234-240.

${ }^{16}$ Gourevitch, Le triangle hippocratique dans le monde gréco-romain, pp. 350-364, ve malignidad en la insinuación de Tácito; cf. además Marasco, «Medici alla corte dei Cesari», p. 291 y n. 62, donde se recogen los análisis que consideran infundada la versión de Tácito.

17 Tenemos noticia de alguno de sus bienes y también del evergetismo practicado en su isla natal. La casa que poseyó parece haber estado situada en el terreno del actual Hospital Militar de Roma, cf. CIL XV 7544, y para la fístula de plomo que la identifica cf. W. Eck, «Die fistulae aquariae der Stadt Rom: Zur Einfluss des sozialen Status auf administratives Handeln», en Epigrafia e ordine senatorio, Roma, 1982, vol. I, pp. 197-225, esp. pp. 201, 204 y 222; además W. Eck, LTUR 2, 1995, p. 182. En CIL VI 8905, ILS 1840 tenemos también alguna información sobre los esclavos de Gayo Estertinio.

${ }^{18}$ Cf. R. Wolters, «C. Stertinius Xenophon von Kos und die Grabinschrift des Trimalchio», Hermes 127, 1999, pp. 47-60; E. Kind, s. u. «Stertinius Xenophon», RE III A 2, 1929, cols. 2450-2451; Herzog, «Nikias und Xenophon von Kos...», pp. 216-247. Cf. supra n. 4. 
Para el segundo de los problemas, el montante de la herencia, el hecho de un error de copia por incomprensión de las siglas $H S$ y del numeral parece probado. En el caso de la lectura que ha dado origen al praenomen es evidente que ninguna de las alternativas textuales parece satisfactoria, aunque una de ellas resultaría posible, pero probablemente también corrupta, como hemos visto, ya que podría explicar los errores de las demás. Queda, pues, un margen razonable de duda sobre el contenido real del texto de Plinio, para no tener que admitir forzosamente el praenomen $Q$. del Stertinius que es citado junto a otros médicos famosos, que no llevan esta precisión en ningún caso.

En suma, creemos que en el texto pliniano el Estertinio mencionado sería el famoso médico de Claudio, que se añadía a la relativamente larga nómina, recogida en el pasaje, de médicos que no necesitaban presentación dada su notoriedad, y del que, además, no cabe duda de que, vista su carrera, debían correr un número importante de anécdotas, entre las cuales Plinio parece haber preferido y elegido tan sólo una. 